\title{
Vibrational signatures of gaseous Meisenheimer complexes bonded at carbon and nitrogen
}

\author{
Barbara Chiavarino, Maria Elisa Crestoni, Simonetta Fornarini* \\ Dipartimento di Chimica e Tecnologie del Farmaco \\ Università di Roma "La Sapienza", P.le A. Moro 5, 00185, Roma (Italy) \\ Fax: (+39) 06-4991-3602 \\ E-mail: Simonetta.fornarini@uniroma1.it
}

Dedicated to Professor Jose Riveros honoring his contribution to gas phase ion chemistry

Keywords : anionic sigma complexes, structure elucidation, IRMPD spectroscopy, photodissociation, FT-ICR mass spectrometry

ABSTRACT: Anionic adducts of 1,3,5-trinitrobenzene (TNB) with deprotonated pyrrolidine (Pyr), imidazole $(\mathrm{Im})$, acetone $(\mathrm{Ac})$ and acetylacetone (Acac) have been delivered into the gas phase by electrospray ionization. The so-formed ions, TNB-Nu' ( $\mathrm{Nu}=\mathrm{Pyr}, \mathrm{Im}, \mathrm{Ac}$, Acac), have been interrogated by IRMPD spectroscopy in the fingerprint range. DFT calculations at B3LYP/6-311++G(d,p) level have been performed for a survey of candidate structures. All adducts conform to anionic $\sigma$-complexes (Meisenheimer complexes). The symmetric stretching modes of the nitro groups yield a dominant vibrational signature at $1200-1250 \mathrm{~cm}^{-1}$, the red-shift with respect to the degenerate frequency of $1367 \mathrm{~cm}^{-1}$ in neutral TNB reflecting the extent of negative charge delocalization. The enol complexes TNB-Ac and TNB-Acac are largely represented by C-bonded species.

Highlights

1. Anionic complexes of 1,3,5 trinitrobenzene are characterized in the gas phase.

2. IRMPD spectroscopy points to a covalent Meisenheimer complex structure.

3. Quantum chemical calculations are employed to assign experimental vibrations.

4. Carbon versus oxygen attack by enolate anions is discussed.

Graphical abstract

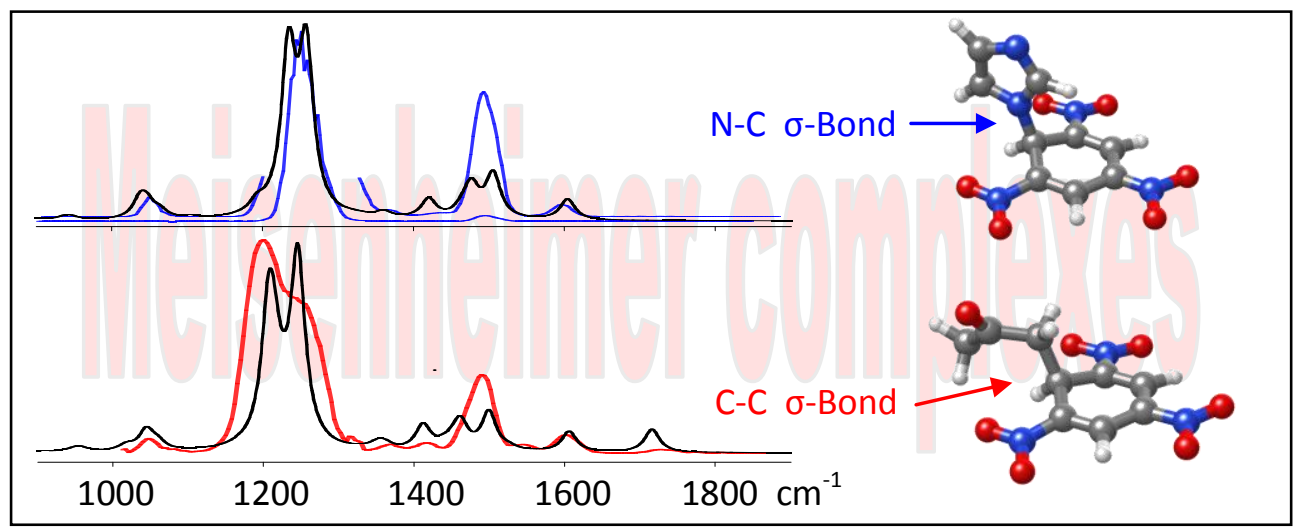




\section{Introduction}

The presence of electron withdrawing substituents on an aromatic ring renders this intrinsically electron rich system susceptible to react with nucleophiles. This interaction is at the basis of the well recognized aromatic nucleophilic substitutions occurring by displacement of a leaving group and representing a class of reactions of fundamental importance in organic chemistry [1]. For pioneering work on aromatic nucleophilic substitution by anionic nucleophiles in the gas phase we are indebted to Josè Riveros and his coworkers [2] who laid the foundations for a wealth of mechanistic studies about anion-arene reactions in an isolated state, extensively surveyed in comprehensive reviews [3-6]. Mechanistic facets have thus emerged that either are peculiar of the gaseous environment, such as the role of multistep reaction sequences occurring within the isolated ion-molecule complex [7-10], or disclose reactivity features typical of $S_{N} A r$ reactions in solution, for example the operation of the so-called element effect governing the leaving group ability of the halide series [11]. The key intermediate in $S_{N} A r$ reactions is an anionic $\sigma$ complex, or Meisenheimer complex. A variety of anion-arene adducts observed in the gas phase are reported to conform to this structure although the structural evidence, typically based on collision induced dissociation (CID) experiments, is indirect $[3,10,12-13]$. However, vibrational spectroscopic information obtained by IR multiple photon dissociation (IRMPD) spectroscopy [14-16] has provided unambiguous indication about the $\sigma$-complex structure of anionic adducts of 1,3,5-trinitrobenzene (TNB) [17-20]. Hydroxide and alkoxide adducts (TNB-OR', with $\mathrm{R}=\mathrm{H}, \mathrm{CH}_{3}$ and $\mathrm{C}_{2} \mathrm{H}_{5}$ ) are covalently bound species with a fully developed $\mathrm{sp}^{3}$ carbon at a formerly unsubstituted ring position [17-18]. Conversely, the halide series presents a transition from a covalent fluoride adduct, TNB-F', to a weak $\sigma$-binding motif for the heavier halide adducts (TNB-X, with $\mathrm{X}=\mathrm{Cl}, \mathrm{Br}, \mathrm{I}$ ) [18-19]. The three nitro groups are strongly electron-withdrawing and impart a highly electrophilic character as found also in 1,3,5-triazine, another example of symmetrical, electron-deficient arene forming anionic covalent $\sigma$-adducts [21-22]. The interest in polynitro-substituted aromatics stems also from their use as explosives and the formation of anionic adducts has been shown to provide a useful detection method for these hazardous compounds [23-25].

In this contribution we focus on the characterization by IRMPD spectroscopy of gaseous anionic $\sigma$ complexes of TNB with carbon- and nitrogen-centered nucleophiles. The complexes, henceforth indicated as TNB-Nu' where $\mathrm{Nu}$ is deprotonated imidazole $(\mathrm{Im})$, deprotonated pyrrolidine (Pyr) and the enolate anions of acetone (Ac) and acetylacetone (Acac), are obtained by electrospray ionization (ESI) of an acetonitrile solution of selected precursors, as first demonstrated by Danikiewicz [26]. Vibrational features are obtained from the experimental IRMPD spectra and their interpretation supported by density functional theory (DFT) ab initio calculations.

\section{Experimental methods}

\subsection{Mass spectrometry and IRMPD spectroscopy}

TNB-Im ${ }^{-}$, TNB-Pyr ${ }^{-}$and TNB-Acac complexes were obtained by ESI in negative ion mode from an acetonitrile solution containing TNB and the selected nucleophile, either imidazole, pyrrolidine or acetylacetone, respectively. In the case of the TNB-Acac adduct, the solution was basified adding $\mathrm{NH}_{3}$. The TNB-Ac complex was obtained from a basic $\left(\mathrm{NH}_{3}\right)$ solution of TNB in $\mathrm{CH}_{3} \mathrm{CN} /$ acetone (1:1). All chemicals were commercial products and used as received.

IRMPD experiments were performed at the European CLIO (Centre Laser Infrarouge d'Orsay) FEL (free electron laser) facility, where the FEL beamline is coupled to a modified commercial 7 T Fourier transform 
ion cyclotron resonance (FT-ICR) mass spectrometer (Bruker, Apex Qe) [27-28]. For the present study the FEL was operated at an electron energy of 42 and $45 \mathrm{MeV}$ to inspect the frequency region of interest (900$\left.2000 \mathrm{~cm}^{-1}\right)$. The laser beam is delivered in $9 \mu$ s macropulses at a repetition rate of $25 \mathrm{~Hz}$. Each macropulse comprises 500 micropulses lasting a few picoseconds and separated by $16 \mathrm{~ns}$. The laser power was ca. 0.9 $1 \mathrm{~W}$ during each run. The ions to be submitted to IRMPD experiments were generated by an Apollo II ESI source and sample solutions were infused at a typical rate of 2-3 $\mu \mathrm{L} \mathrm{min}^{-1}$. The anionic complexes were mass selected in the quadrupole and thermalized by multiple collisions with argon in a linear hexapole, prior to their transfer into the ICR cell [29]. The isolated charged complexes were then irradiated for $200 \mathrm{~ms}$ to $2 \mathrm{~s}$ with the IR FEL light, after which the mass spectrum was recorded. IRMPD spectra are obtained by plotting the photofragmentation yield $R\left(R=-\ln \left[I_{\text {parent }} /\left(I_{\text {parent }}+\Sigma I_{\text {fragment }}\right)\right]\right)$, where $I_{\text {parent }}$ and $I_{\text {fragment }}$ are the integrated abundancies of the mass peaks of the precursor and of the fragment ions, respectively) as a function of the wavenumber of the IR radiation.

\subsection{Computational details}

DFT calculations were performed using the Spartan'16 program package. A preliminary inquiry of isomeric structures and conformers was run at the B3LYP/6-31G(d) level of theory. The most stable geometries were then further optimized at $B 3 L Y P / 6-311++G(d, p)$ level. Frequency analysis, carried out at the same level, confirms the obtained geometries to lie in an energy minimum and provides the respective IR spectra. Hybrid DFT methods, such as B3LYP, are known to perform well in yielding infrared frequencies and intensities, provided a scaling factor is adopted to account for anharmonicity [15]. Computed IR absorption spectra are plotted using a scaling factor of 0.976 and assuming a Gaussian shape with a full width at half maximum of $20 \mathrm{~cm}^{-1}$ to display a pattern directly comparable with the experimental IRMPD spectrum. Reported relative energies are enthalpies at $298 \mathrm{~K}$.

\section{Results and discussion}

\subsection{Meisenheimer complexes bonded at nitrogen}

When the intensely red-colored solution of TNB and pyrrolidine is analyzed in ESI negative-ion mode, the major ionic species at $\mathrm{m} / \mathrm{z} 283$ is consistent with the formation of a TNB-Pyr complex [TNB- $\left.\mathrm{NC}_{4} \mathrm{H}_{8}\right]^{-}$. The fragmentation of TNB-Pyr upon irradiation by IR photons in resonance with an active vibrational mode leads to a major product ion at $m / z 213$, corresponding to TNB radical anion (TNB ${ }^{-1}$ [Eq. (1 a)]. A second, minor fragment ion at $\mathrm{m} / \mathrm{z} 183$ is due to further loss of NO [Eq. (1b)]. This fragmentation pattern was also observed in CID experiments on negatively charged adducts of TNB with various amines [26].

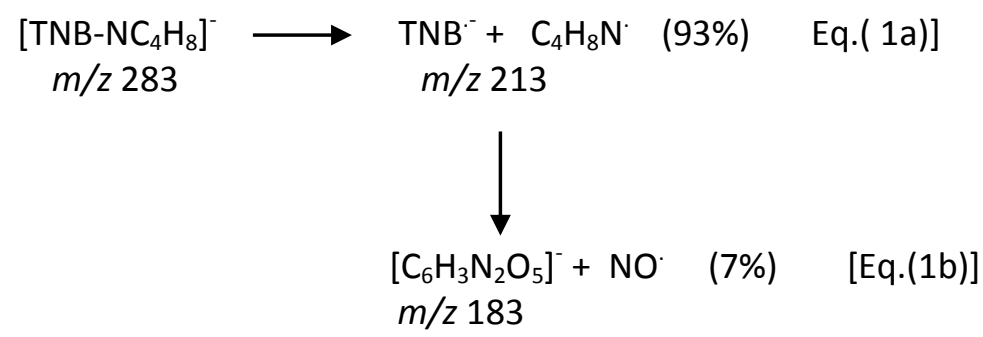

The TNB-Im complex at $m / z 280$ presents a distinct fragmentation pattern, yielding two fragment ions with approximately the same abundance, namely an ion at $m / z 212$, deprotonated TNB formed by loss of 
imidazole [Eq. (2 a)], and an ion at $\mathrm{m} / \mathrm{z} 204$, involving loss of dinitrogentrioxide (or combined loss of NO and of $\mathrm{NO}_{2}$ ) [Eq. (2 b)].

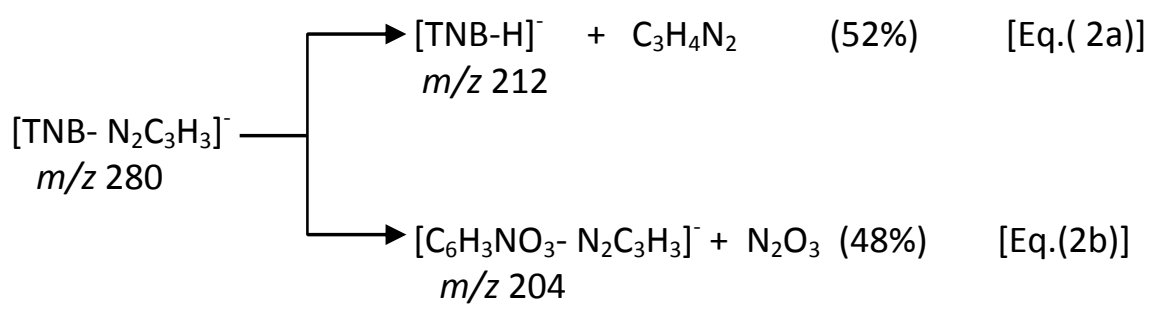

Mass spectrometric analysis has thus shown that both pyrrolidine ( $\left(\mathrm{c}_{4} \mathrm{C}_{8} \mathrm{H}_{8} \mathrm{NH}\right.$ a cyclic secondary amine) and imidazole $\left(\mathrm{C}_{3} \mathrm{H}_{4} \mathrm{~N}_{2}\right.$ powerful heterocyclic nucleophile) yield anionic adducts with TNB. The so-formed TNBPyr ${ }^{-}$and TNB-Im complexes formally involve addition of the deprotonated nucleophiles. However, $\mathrm{S}_{N} \mathrm{Ar}$ reactions between amine nucleophiles and activated aromatics in solution involve a stepwise mechanism occurrig via a zwitterionic intermediate which is deprotonated to give the anionic adducts [30]. In this report the focus is on the actual structural features of the anionic species, once delivered by negative ESI to the gas phase. To this end, IRMPD spectra have been recorded and are plotted in panel (a) of Figs. 1 and 2 for TNB-Pyr and TNB-Im; , respectively.

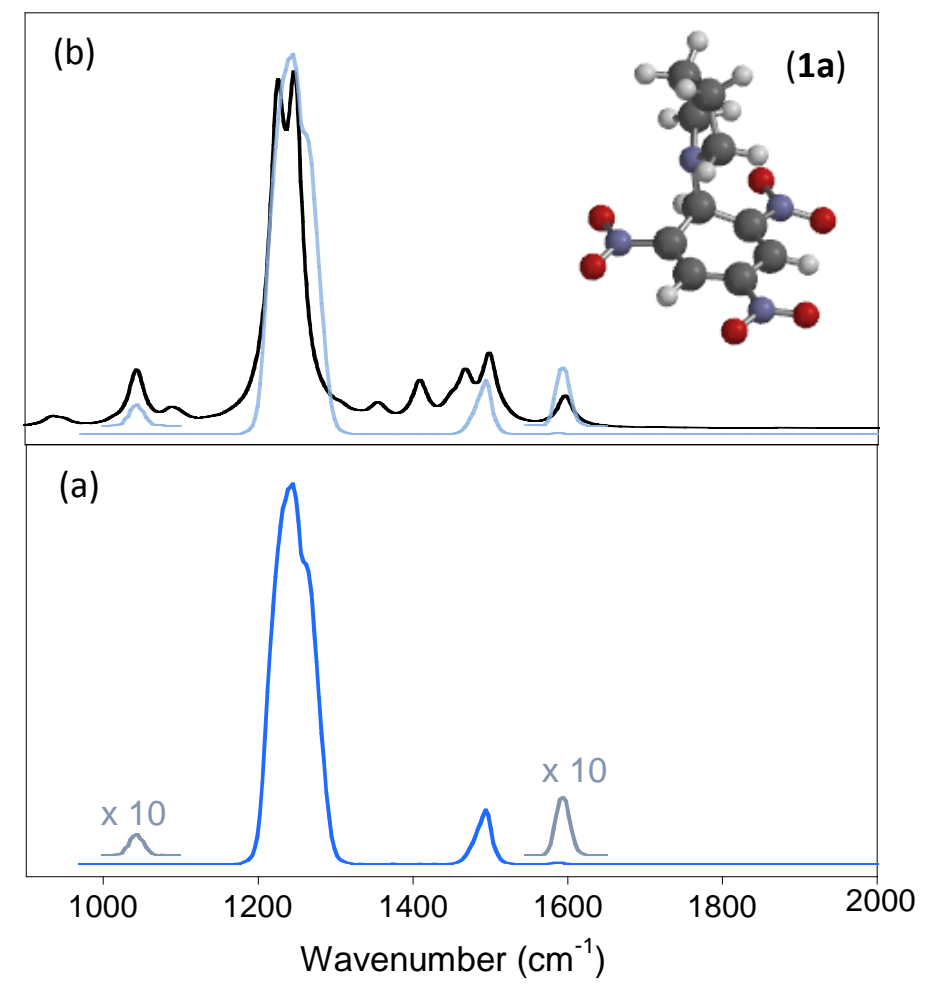

Fig. 1. Experimental IRMPD spectrum of TNB-Pyr ${ }^{-}$ions (a) and computed IR spectrum of 1a (b). 

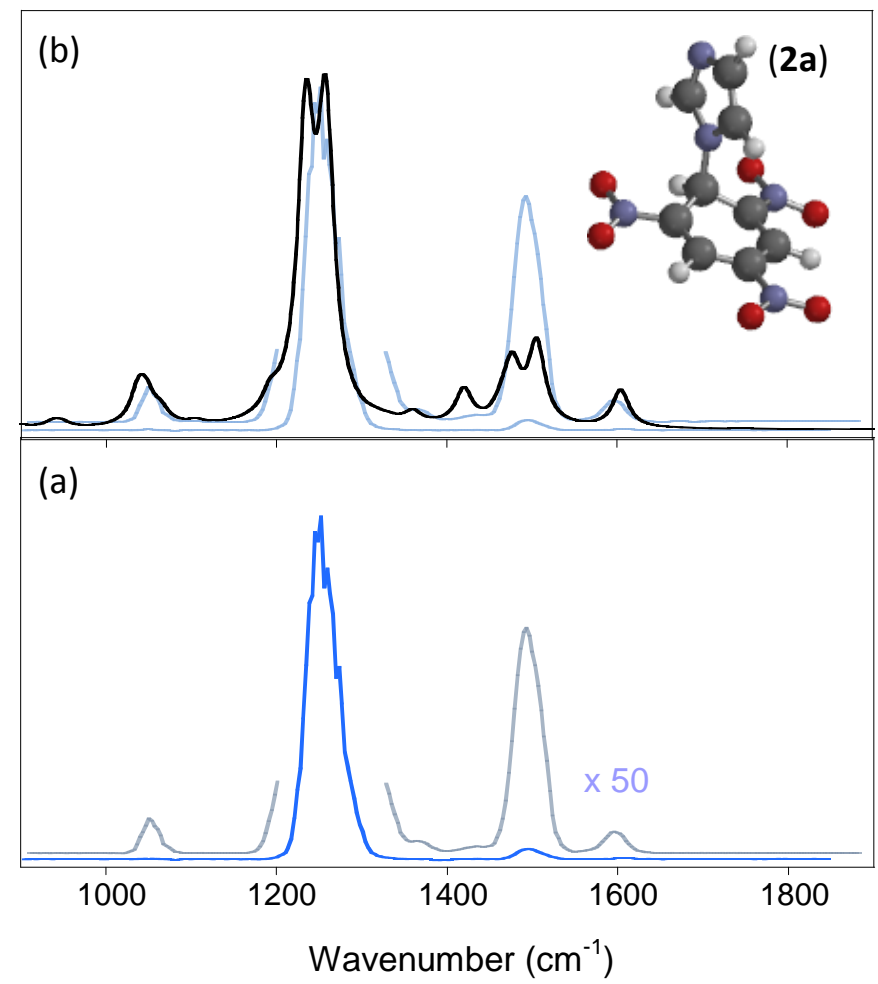

Fig. 2. Experimental IRMPD spectrum of TNB-Im ions (a) and computed IR spectrum of $2 \mathrm{2a}(\mathrm{b})$.

The IRMPD spectra of TNB-Pyr and TNB-Im ${ }^{-}$are remarkably similar, both characterized by a wide absorption centered at ca. $1250 \mathrm{~cm}^{-1}$ and weaker bands at 1042-1050, 1492-1495, and $1596 \mathrm{~cm}^{-1}$ (Tables 1 and 2). One may thus infer that (i) the two ions share a common structure and (ii) modes pertaining to the bound nucleophile present either scant or quite comparable contribution to the IR spectrum. In particular, the wide band at $1250 \mathrm{~cm}^{-1}$ represents a highly diagnostic feature, characteristic of a strongly covalent anionic $\sigma$-complex. Indeed, a comparable distinct absorption at $1253 \mathrm{~cm}^{-1}$, assigned to $\mathrm{NO}_{2}$ symmetric stretching, has been reported for example for the methoxide adduct $\mathrm{TNB}^{-\mathrm{OCH}_{3}}{ }^{-}$[18].

As commonly practiced, mode assignment of the IRMPD bands and structural insight are gained by comparing the experimental spectrum with the calculated IR spectrum of candidate structure(s). Both TNBPyr and TNB-Im ${ }^{-}$are found to conform to stable anionic $\sigma$-complexes characterized by a covalent C-N bond engaging an unsubstituted carbon of TNB. The structures of TNB-Pyr ${ }^{-}$(1a) and of TNB-Im ${ }^{-}$(2a) are depicted in panel (b) of Figs. 1 and 2, respectively, while the Supplementary Data (SD) presents a list of thermodynamic data (Table S1) and Cartesian coordinates (Table S2) of all calculated structures. Interestingly, TNB-Pyr (1a) presents an approximately perpendicular arrangement of the pyrrolidine unit with respect to the TNB ring with a $\mathrm{C}_{\text {pyr }} \mathrm{NC}_{\text {ipso }} \mathrm{C}_{\text {para }}$ dihedral angle of $15^{\circ}$. The optimized geometry of a "parallel" rotamer $\mathbf{1 b}\left(\mathrm{C}_{\text {pyr }} \mathrm{NC}_{\text {ipso }} \mathrm{C}_{\text {para }}\right.$ dihedral angle of $\left.119^{\circ}\right)$ is shown in Fig. $\mathrm{S} 1$ in the SD. It lies $9 \mathrm{~kJ} \mathrm{~mol}^{-1}$ higher in energy relative to $\mathbf{1 a}$, as shown in Table S1. The IR spectrum of $\mathbf{1 b}$ (Fig. S1) is quite similar to the one of 1a, reported in Fig. 1(b). The experimental IRMPD bands are then interpreted by referring to $\mathbf{1 a}$, as shown in detail in Table 1 listing the vibrational data. 
Table 1: Experimental IRMPD bands observed for TNB-Pyr complex and computed vibrational modes for the TNB-Pyr (1a) rotamer.

\begin{tabular}{|c|c|c|}
\hline Experimental IRMPD ${ }^{a}$ & Calculated $^{\mathrm{a}, \mathrm{b}}$ & Vibrational mode \\
\hline 1042 & 1043(211), 1045 (122) & $\mathrm{CH}$ bending (in plane) \\
\hline- & $1088(64)$ & $\mathrm{C}-\mathrm{N}(\mathrm{Pyr})$ stretch \\
\hline $1240-1260$ & 1225 (1679), 1248 (1755) & $\mathrm{NO}_{2}$ symm stretch \\
\hline- & 1353(52), $1358(51)$ & Wagg $\mathrm{CH}_{2}$ (Pyr) \\
\hline- & $1409(244)$ & $\mathrm{NO}_{2}$ asymm stretch and $\mathrm{CC}_{\mathrm{TNB}}$ stretch \\
\hline 1495 & $1468(254), 1499$ (395) & $\mathrm{NO}_{2}$ asymm stretch and $\mathrm{C}-\mathrm{N}\left(\mathrm{NO}_{2}\right)$ stretch \\
\hline 1596 & $1597(183)$ & $\mathrm{CC}_{\mathrm{TNB}}$ stretch \\
\hline
\end{tabular}

Table 2: Experimental IRMPD bands observed for TNB-Im complex and computed vibrational modes for the TNB-Im- $(2 \mathrm{a})$ rotamer.

\begin{tabular}{lll} 
Experimental IRMPD $^{\mathrm{a}}$ & Calculated $^{\mathrm{a}, \mathrm{b}}$ & Vibrational mode \\
\hline 1050 & $1038(200), 1047(130), 1069(80)$ & $\mathrm{CH}$ bending (in plane) \\
& $1198(107)$ & $\mathrm{C}-\mathrm{N}(\mathrm{Im})$ stretch \\
1250 & $1235(1683), 1258(1697)$ & $\mathrm{NO}_{2}$ symm stretch \\
1365 & $1360(67)$ & $\mathrm{C}-\mathrm{N}\left(\mathrm{NO}_{2}\right)$ stretch \\
1429 & $1419(200)$ & $\mathrm{CC}_{\mathrm{TNB}}$ stretch \\
1492 & $1465.1(61), 1475(151), 1478(205)$, & $\mathrm{NO}_{2}$ asymm stretch, $\mathrm{C}-\mathrm{N}(\mathrm{Im})$ stretch, \\
& $1505(463)$ & $\mathrm{NO}_{2}$ asymm stretch and $\mathrm{CC} C_{\mathrm{TNB}}$ stretch \\
1596 & $1604.9(218)$ & $\mathrm{CC}_{\mathrm{TNB}}$ stretch
\end{tabular}

${ }^{\text {a }}$ Frequency in $\mathrm{cm}^{-1}$

${ }^{b}$ Calculated vibrational modes at the B3LYP/6-311++G(d,p) level of theory. The computed intensities ( $k m$ $\mathrm{mol}^{-1}$ ) are given in parenthesis. Bands with intensity lower than $40 \mathrm{~km} \mathrm{~mol}^{-1}$ are not included.

The $2 \mathrm{a}$ geometry of the TNB-Im complex is characterized by a perpendicular orientation of the two rings. In this case a parallel type of arrangement does not correspond to a local minimum, rather evolving to $2 \mathrm{a}$ on geometry optimization. A rotamer can however be envisioned with the imidazole aza group pointing away from the TNB ring (2b). Energies and IR spectra of the two isomers are practically the same as shown in Fig. S2 and Table S1 in the SD. Furthermore, a TNB-Im complex involving attack at a nitro-substituted carbon, however kinetically unfavourable, has been taken into consideration. Upon geometry optimization, the starting geometry evolves by $\mathrm{C}-\mathrm{NO}_{2}$ bond cleavage, as already reported for comparable species $[18,20]$. The $\mathrm{NO}_{2}$ group then binds to an adjacent carbon and structure $\mathbf{2 c}$ is finally reached, corresponding to an $\mathrm{NO}_{2}{ }^{-} \sigma$-complex with two $o, p$-nitro groups and an Im group in ortho position to the tetrahedral carbon (see 
structure depicted in the SD). The calculated IR spectrum, also shown in Fig. 2, does not account well for the experimental IRMPD spectrum.

Because the vibrational spectra of TNB-Pyr and TNB-Im complexes are remarkably similar they are discussed together. In order to appreciate fine differences one may refer to Tables 1 and 2. As stated earlier, the dominant band centered at $1250 \mathrm{~cm}^{-1}$ is due to the highly active resonances involving $\mathrm{NO}_{2}$ symmetric vibrations. The corresponding resonance in neutral TNB is found at $1367 \mathrm{~cm}^{-1}$ and the pronounced red-shift in the anionic complex is ascribed to charge delocalization onto the nitro groups [19]. They become non-equivalent and the $\mathrm{N}-\mathrm{O}$ bonds are weakened, thus accounting for band splitting and shifting to lower frequency. Where only a 'weakly' covalent $\sigma$-character is present, as in the heavier halide TNB complexes, the effect is less pronounced and in fact these complexes present a rather narrow band at higher frequency (1332-1345 $\mathrm{cm}^{-1}$ ) indicating a scantly perturbed TNB unit [19]. Thus, the absorption at $1250 \mathrm{~cm}^{-1}$ can be regarded as clear signature of the anionic (Meisenheimer) $\sigma$-complex.

For comparison purposes, one may note that when a nitro group is in direct conjugative interaction with a negative charge placed in benzylic position as in deprotonated 2,4-dinitrotoluene, the red shift of the $\mathrm{NO}_{2}$ symmetric stretching mode is even more pronounced yielding a strong IRMPD band at $1180 \mathrm{~cm}^{-1}$ [31]. Resonance structures delocalizing the negative charge and weakening the NO bond are also responsible for the comparatively low frequency of the $\mathrm{NO}_{2}$ symmetric stretch observed in the IRMPD spectrum of the nitrobenzene radical anion [32].

The band at $1042-1050 \mathrm{~cm}^{-1}$ is associated to the TNB CH in plane bending. In the $1400-1500 \mathrm{~cm}^{-1}$ wavenumber range one expects $\mathrm{NO}_{2}$ asymmetric stretchings and TNB ring deformation modes. Only one band is observed at $1492-1495 \mathrm{~cm}^{-1}$. Modes of lower intensity are apparently missing, likely due to the nonlinear character of the IRMPD process [14-16]. The band at $1596 \mathrm{~cm}^{-1}$ is associated to TNB C-C stretching vibrations.

\subsection{Meisenheimer complexes bonded at carbon}

Sampling of TNB-Ac complex ( $\mathrm{m} / \mathrm{z} 270$ ) by IRMPD spectroscopy has shown photofragmentation occurring along two channels. The major one is associated with loss of $\mathrm{HNO}_{2}$ giving an ion at $\mathrm{m} / \mathrm{z} 223$ [Eq. (3 a)]. This product ion has been ascribed the structure of a nitrobenzyl-substituted acetonyl anion when the TNB-Acprecursor is obtained using APCI mass spectrometry [24]. The second channel corresponds to the loss of neutral acetone, to generate the deprotonated TNB at $m / z$ 212. [Eq. (3 b)]. Nitrite anion $\left(\mathrm{NO}_{2}\right)^{-}(\mathrm{m} / z$ 46) and enolate anion of acetone $(\mathrm{m} / \mathrm{z} 57)$, fragments found in the CID spectra of the $\sigma$-adduct of 1,3 dinitrobenzene with acetone, were not observed in the current IRMPD experiments in line with the higher acidity of TNB [26].

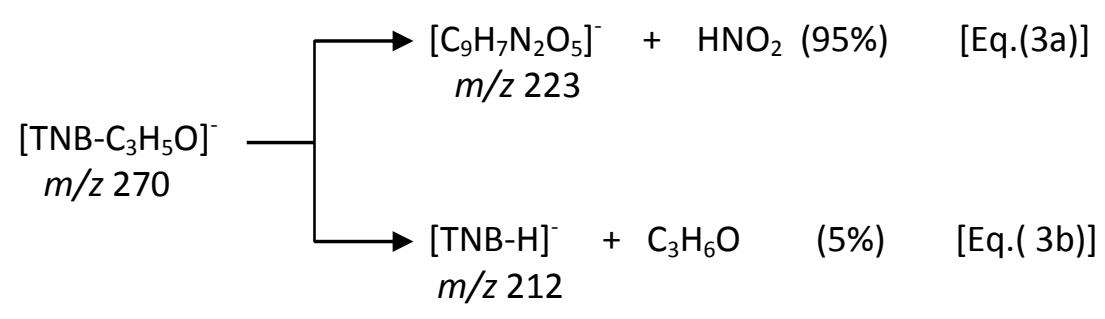




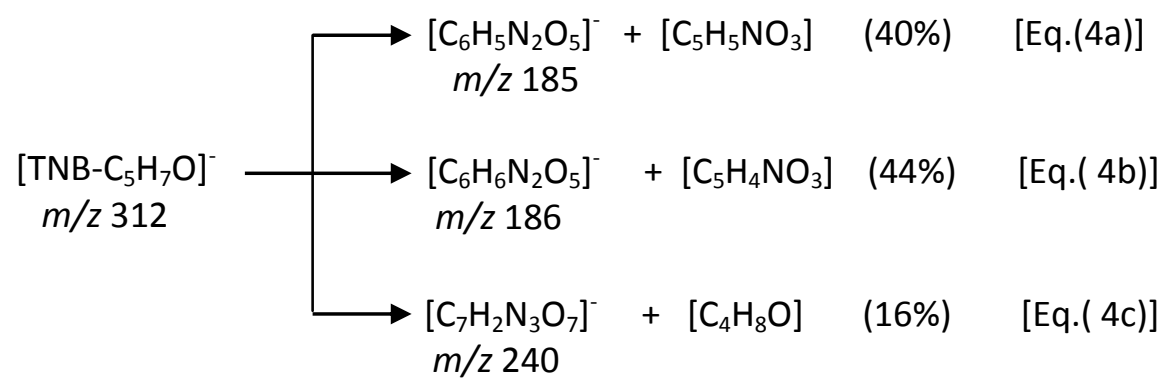

The TNB-Acac' complex ( $\mathrm{m} / \mathrm{z}$ 312) undergoes photofragmentation yielding ions at $\mathrm{m} / \mathrm{z} \quad 185,186$ and 240 according to processes involving extensive bond breaking and rearrangement [Eq. (4 a-c)].

The IRMPD spectra of TNB-Ac' and TNB-Acac are reported in Figs. 3 and 4 (panel (a)), respectively.

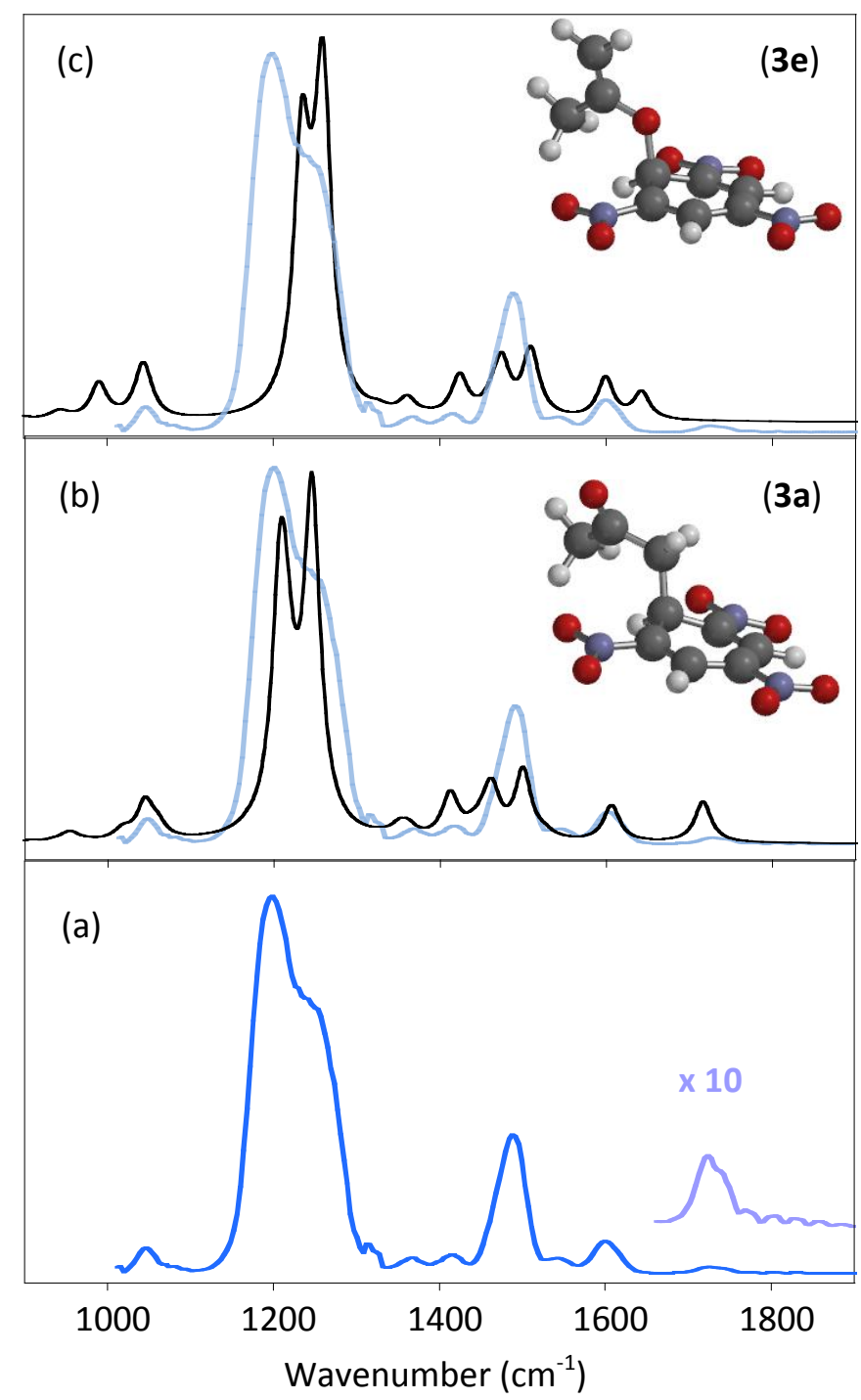

Fig. 3. Experimental IRMPD spectrum of TNB-Ac ions (a) and computed IR spectra of $\mathbf{3 a}$ (b) and $\mathbf{3 e}$ (c). 
The general pattern of the spectra shows remarkable similarity with the spectra of TNB-Pyr and TNB-Im;, supporting a common structure with characteristic signatures dominated by the TNB component. Thus, the strong, wide band at $1196-1240 \mathrm{~cm}^{-1}$ is accompanied by weaker features at 1046-1050, 1490, 1596-1600 $\mathrm{cm}^{-1}$. An additional band recorded at $1718-1744 \mathrm{~cm}^{-1}$ is in the range expected for a carbonyl $\mathrm{C}=0$ stretching frequency, therefore a distinct signature of the nucleophile. At variance with the nitrogen nucleophiles used, enolate anions present potential ambident reactivity, involving either the carbon or the oxygen nucleophilic center. Systematic studies have revealed a range of selectivities varying from exclusive reaction at oxygen to sole attack at carbon in the reaction enolate anions with a variety of gaseous electrophiles $[3,5,33]$. In the present study, the sampled TNB-Ac ${ }^{-}$and TNB-Acac are formed in solution and the complexes are likely kinetically trapped in their original structure.

Isomeric species have been examined computationally, obtained by either carbon or oxygen attack by the acetone enolate ion. Fig. 3 (b) shows the structure and IR spectrum of $\mathbf{3 a}$, a carbon-bonded TNB-Ac' complex. In this species the $\mathrm{CH}_{3} \mathrm{COCH}_{2}$ group is anti-oriented with respect to the TNB ring. A syn-type geometry in $\mathbf{3 b}$ yields a conformer only slightly higher in energy (at $6 \mathrm{~kJ} \mathrm{~mol}^{-1}$ ) shown in Fig. $\mathrm{S} 3$ together with 3c, another anti-type conformer at $9 \mathrm{~kJ} \mathrm{~mol}^{-1}$ relative energy. The calculated IR spectra, also shown in Fig. 3(b) and Fig. S3(b-c), are quite comparable, both in terms of frequencies and intensities, and, as shown by the superimposed IRMPD spectrum, account well for the experimentally observed features. A detailed matching and mode assignment is illustrated in Table 3.

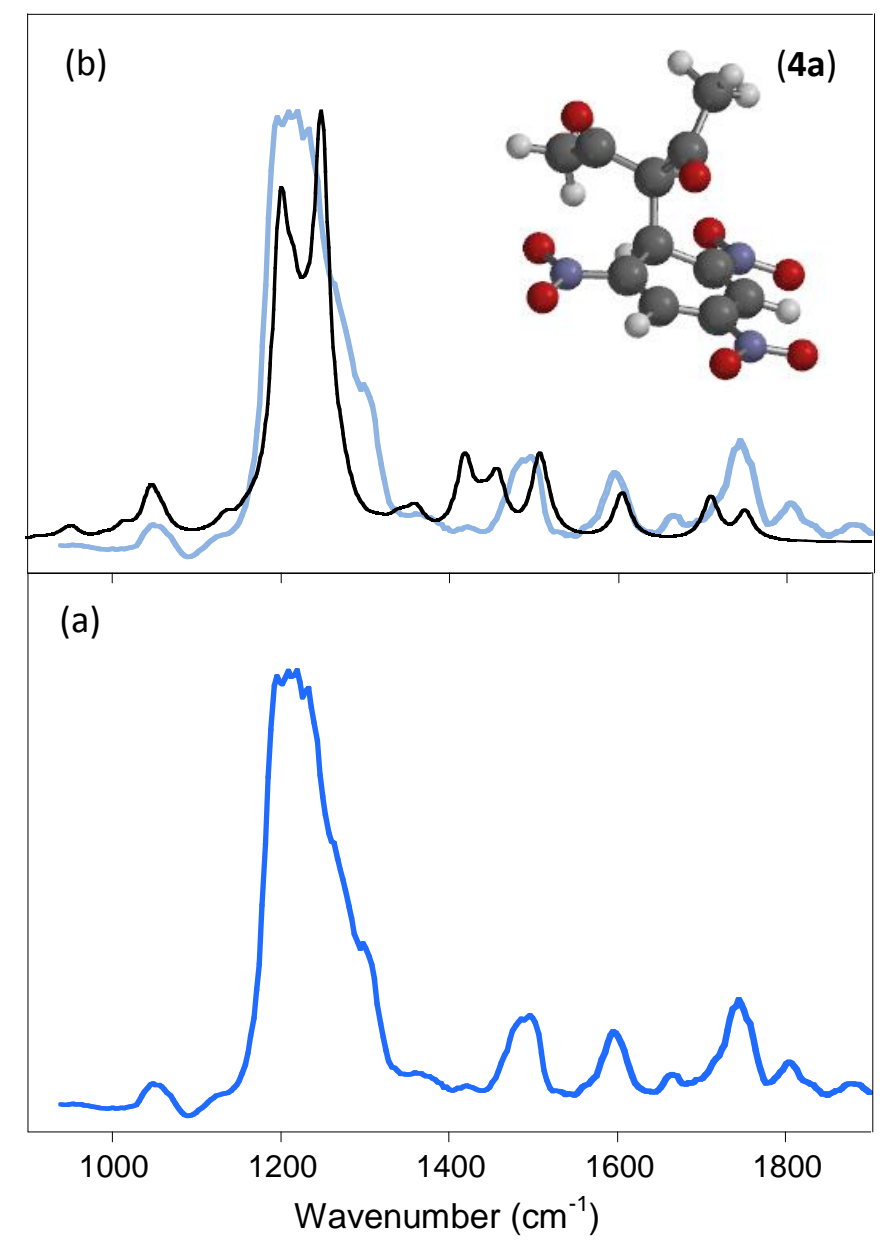

Fig. 4. Experimental IRMPD spectrum of TNB-Acac ions (a) and computed IR spectrum of 4 a (b). 
Table 3: Experimental IRMPD bands observed for TNB-Ac complex and computed vibrational modes for the TNB-Ac' (3a) isomer.

\begin{tabular}{|c|c|c|}
\hline Experimental IRMPD ${ }^{\mathrm{a}}$ & Calculated ${ }^{a, b}$ & Vibrational mode \\
\hline 1046 & $1045(229), 1060(79)$ & $\mathrm{CH}$ bending (in plane), $\mathrm{CH}_{3}$ Wag \\
\hline 1196 & $1207(1370), 1215(557), 1228$ (100) & $\begin{array}{l}\mathrm{NO}_{2} \text { symm stretch, } \mathrm{CH} \text { bending (in } \\
\text { plane), }\end{array}$ \\
\hline 1230 & $1246(2049)$ & $\mathrm{NO}_{2}$ symm stretch \\
\hline 1365 & 1351(61),1361(59). & $\mathrm{CH}_{3}$ umbrella , $\mathrm{C}-\mathrm{N}$ stretch \\
\hline 1414 & $1412(260)$ & $\begin{array}{l}\mathrm{NO}_{2} \text { asymm stretch and } \mathrm{CC}_{\mathrm{TNB}} \\
\text { stretch }\end{array}$ \\
\hline 1488 & 1454 (77), $1462(271), 1500$ (419) & $\begin{array}{l}\mathrm{CH}_{3} \text { Rock, } \mathrm{NO}_{2} \text { asymm stretch, } \mathrm{CC}_{\mathrm{TNB}} \\
\text { stretch }\end{array}$ \\
\hline 1600 & $1606(222)$ & $\mathrm{CC}_{\mathrm{TNB}}$ stretch \\
\hline 1718 & $1716(252)$ & CO stretch \\
\hline
\end{tabular}

${ }^{a}$ Frequency in $\mathrm{cm}^{-1}$

${ }^{b}$ Calculated vibrational modes at the B3LYP/6-311++G(d,p) level of theory. The computed intensities (km $\mathrm{mol}^{-1}$ ) are given in parenthesis. Bands with intensity lower than $40 \mathrm{~km} \mathrm{~mol}^{-1}$ are not included.

Table 4: Experimental IRMPD bands observed for TNB-Acac complex and computed vibrational modes for the TNB-Acac ${ }^{-}(4 a)$ isomer.

\begin{tabular}{lll} 
Experimental IRMPD & Calculated & Vibrational mode \\
\hline 1050 & $1047(203), 1060(72)$ & $\mathrm{CH}_{\text {bending }}$ (in plane), $\mathrm{CH}_{3}$ Wag \\
1128 & $1135(58)$ & $\mathrm{CC}_{\text {Acac }}$ stretch, $\mathrm{CH}_{3}$ Wag \\
$1196-1240$ & $1200(1274), 1216(567), 1230(359)$, & $\mathrm{NO}_{2}$ symm stretch, $\mathrm{CH}$ bending (in \\
& $1249(1753)$ & plane) \\
1198 & $1274(116)$ & $\mathrm{CH}$ bending \\
1367 & $1360(82)$ & $\mathrm{CH}_{3}$ Umbrella, $\mathrm{C}-\mathrm{N}$ stretch, $\mathrm{CH}$ bending \\
1420 & $1419(333)$ & $\mathrm{NO}_{2}$ asymm stretch $\mathrm{CC}_{\text {TNB }}$ stretch \\
1491 & $1440(79), 1458(248), 1508(374)$ & $\mathrm{Rock}_{3}, \mathrm{NO}_{2}$ asymm stretch $\mathrm{CC}_{\text {TNB }}$ \\
& & stretch \\
1596 & $1606(218)$ & $\mathrm{CC}_{\text {TNB }}$ stretch \\
1744 & $1711(200), 1751(132)$ & $\mathrm{CO}$ stretch
\end{tabular}

${ }^{a}$ Frequency in $\mathrm{cm}^{-1}$

${ }^{b}$ Calculated vibrational modes at the B3LYP/6-311++G(d,p) level of theory. The computed intensities ( $\mathrm{km}$ $\mathrm{mol}^{-1}$ ) are given in parenthesis. Bands with intensity lower than $40 \mathrm{~km} \mathrm{~mol}^{-1}$ are not included.

The $\mathrm{CH}_{3} \mathrm{COCH}_{2}$ group may conceivably rearrange to an enol structure and the ensuing $\mathrm{CH}_{3} \mathrm{C}(\mathrm{OH})=\mathrm{CH}_{2}$ group is represented in TNB-Ac isomer $\mathbf{3} \mathbf{d}$ (at $33 \mathrm{~kJ} \mathrm{~mol}^{-1}$ relative energy) whose structure and IR spectrum are depicted in Fig. S3(d). The IR spectrum is once again quite similar to the ones of the former species, only differing for the absence of the $\mathrm{C}=\mathrm{O}$ stretching band and the presence of a $\mathrm{COH}$ bending coupled to $\mathrm{C}-\mathrm{C}$ 
stretching mode at $1670 \mathrm{~cm}^{-1}$. Finally, an oxygen-bonded isomer 3 e (Fig. 3(c)) has been calculated to lie at $84 \mathrm{~kJ} \mathrm{~mol}^{-1}$ relative energy. However, its IR spectrum, characterized by symmetric NO2 stretching modes at somewhat higher frequency than experimentally observed, by the $\mathrm{C}=\mathrm{C}$ stretch of the $\mathrm{CH}_{3} \mathrm{C}(\mathrm{OH})=\mathrm{CH}_{2}$ group calculated at $1643 \mathrm{~cm}^{-1}$ and the obvious lack of a $\mathrm{C}=\mathrm{O}$ stretch matching the experimental band at $1718 \mathrm{~cm}^{-1}$, does not support a significant presence of this isomer. In view of the wide width of the band at 1196-1240 $\mathrm{cm}^{-1}$ it is possible, though, that multiple conformers may contribute to the sampled ion population. For example $3 \mathrm{~d}$ presents a wider separation of the $\mathrm{NO}_{2}$ symmetric stretch bands though still centered relative to the experimental absorption feature. However its presence, as well as that of $\mathbf{3 e}$ is unlikely to be significant due also to their considerably higher energy content.

The acetylacetone enolate is stabilized by two carbonyl groups and carbon bonding at the highly activated methylene position has been taken into consideration to account for the structure of the assayed TNBAcac complex. The $\mathbf{4 a}$ conformer depicted in Fig. 4(b) presents one acetyl group in syn-like orientation relative to the TNB ring while the second one is anti-like oriented with a dihedral angle measuring the mutual orientation of the two carbonyl groups of $71^{\circ}$. The IR spectrum is in fair agreement with the experimental IR spectrum, as also testified by the vibrational frequencies listed in Table 4. Once again the major features in the observed portion of the IR spectrum are associated to the TNB moiety of the TNB-

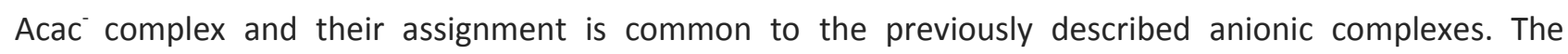
characteristic signature of TNB-Acac is the carbonyl stretch band at $1744 \mathrm{~cm}^{-1}$ in the IRMPD spectrum. This relatively wide band is probably a convolution of the two $\mathrm{C}=\mathrm{O}$ stretch resonances calculated at 1751 and $1711 \mathrm{~cm}^{-1}$. Because the vibrational spectrum is dominated by the TNB unit bearing the negative charge, conformational variability in the Acac group is not expected to produce significant changes. Indeed, conformer $\mathbf{4} \mathbf{b}$, differing for the rotation of the two acetyl groups and lying $14 \mathrm{~kJ} \mathrm{~mol}^{-1}$ higher in energy relative to $4 \mathbf{a}$, presents quite comparable spectral features (Fig.S4(b)). Tautomerization of the Acac group via hydrogen migration from the methine carbon to a carbonyl oxygen yields a stable enol isomer, $4 c$, at -25 $\mathrm{kJ} \mathrm{mol}^{-1}$ relative to $4 \mathrm{a}$, displaying an intramolecular hydrogen bond. As shown in Fig. S4(c) the hydrogen bond weakens the force constant of the remaining carbonyl now absorbing at $1573 \mathrm{~cm}^{-1}$. However, given the relatively weak calculated intensity of this mode and the remaining features of the spectrum sharing similar frequency and intensity with the keto isomer, one cannot exclude a contribution of this species in the sampled ion population. Finally, also a complex involving attack by the less activated methyl carbon of acetylacetone has been conceived, corresponding to isomer $\mathbf{4 d}$ (Fig.S4(d)). Complex $\mathbf{4 d}$ turns out to be more stable than $4 \mathrm{a}$ by $12 \mathrm{~kJ} \mathrm{~mol}^{-1}$, which may be partly attributed to diminished steric congestion at the tetrahedral ring carbon. While its formation will be kinetically disfavored in solution, its IR spectral features are once again largely dominated by the TNB moiety making it difficult to discard its potential contribution.

\section{Conclusions}

Nucleophilic aromatic substitution reactions display a variety of reactivity patterns in the gas phase, depending on the nucleophile, the leaving group and the activation of the aromatic substrate, as recently illustrated in a thorough computational survey [34]. Anionic $\sigma$-complexes may occur as reaction intermediates or transition states. An example of Meisenheimer complex representing a transition state rather than a local energy minimum is reported by Riveros in the unusually fast nucleophilic aromatic displacement reaction of nitrobenzene with fluoride ion, studied by FT-ICR mass spectrometry [35]. The three nitro groups of TNB provide considerable stabilization on Meisenheimer complexes and the selected TBN-Nu' ( $\mathrm{Nu}=$ Pyr, Im, Ac, Acac) adducts formed in solution and assayed in the gas phase all conform to an anionic covalent structure. This conclusion is supported by the vibrational signatures recorded in the 
fingerprint range of the IR spectrum and by DFT computational data providing geometries, energies and IR spectra of plausible stable structures. The covalent $\sigma$-complex structure is characterized in particular by the band associated to strongly active $\mathrm{NO}_{2}$ symmetric stretching modes that are significantly red-shifted relative to neutral TNB. The extent of this shift as well as the separation of the resonances for the nonequivalent ortho and para groups may be considered a reflection of the $\sigma$-covalent character of the complex. The IRMPD bands for the TBN-Nu' ( $\mathrm{Nu}=\mathrm{Pyr}, \mathrm{Im}, \mathrm{Ac}$, Acac) complexes are centered at 1240-1260, $1250,1196-1230$ and $1196-1240 \mathrm{~cm}^{-1}$, respectively. Unfortunately, only a convoluted envelope of the $\mathrm{NO}_{2}$ symmetric stretching modes is experimentally observed. According to the calculations though, the most active resonances pertaining to ortho (at lower frequency) and ortho/para (higher frequency) nitro group symmetric stretching are separated by a gap of $23,23,39$, and $30 \mathrm{~cm}^{-1}$ in the Pyr, Im, Ac, and Acac series of adducts (see computed data in Tables 1-4). These values may be compared with 23 and $26 \mathrm{~cm}^{-1}$ gaps obtained for methoxide and cyanide complexes, respectively $[18,20]$. In marked contrast, the corresponding difference is calculated to be ca $4 \mathrm{~cm}^{-1}$ for the bromide complex, TNB-Br-, characterized by a weakly covalent bond of $\mathrm{Br}$ - with a nearly unperturbed TNB unit. An inference my thus be suggested that the enol anions impart an even more strongly covalent $\sigma$-complex character, relative to other tested nucleophiles. TBN-Nu' complexes with enolate ions conform to carbon-bonded species, in agreement with the presence of a $\mathrm{C}=\mathrm{O}$ stretching signature. The formation of a carbon-bonded adduct of acetone enolate anion with $m$-dinitrobenzene in an ESI source had been proposed on the basis of the common CID pattern with a complex formed in solution [10]. Forthcoming efforts will be devoted to exploit IRMPD spectroscopy to gain insight into the structure of anionic complexes of electron-deficient arenes obtained from reactions conducted in different media.

\section{Acknowledgements}

This work was supported by the Università di Roma La Sapienza and by the European Commission (CLIO project IC021-09) We are grateful to J. M. Ortega, P. Maitre, D. Scuderi, V. Steinmetz and to the technical staff members at the CLIO facility.

\section{Appendix A. Supplementary data}

Supplementary data associated with this article can be found in the online version.

\section{References}

[1] F. Terrier, Modern Nucleophilic Aromatic Substitution, Wiley-VCH Verlag , Weinheim, 2013.

[2] S.M.J. Briscese, J.M. Riveros, Gas Phase Nucleophilic Reactions of Aromatic Systems, J. Am. Chem. Soc.

97 (1975) 230-231.

[3] W. Danikiewicz, M. Zimnicka, Negative Ion Gas-Phase Chemistry of Arenes, Mass Spectrom. Rev. 35

(2016) 123-146.

[4] N.M.M. Nibbering, Highlights of 50 Years of Ionic Reaction Mechanistic Studies, Int. J. Mass Spectrom.

377 (2015) 10-22.

[5] Z. Tian, S.R. Kass, Carbanions in the Gas Phase, Chem. Rev. 113 (2013) 6986-7010.

[6] R. R. Squires, Gas-Phase Carbanion Chemistry, Acc. Chem. Res. 25 (1992) 461-467.

[7] S. Ingemann, N.M.M. Nibbering, S.A. Sullivan, C.H. DePuy, Nucleophilic Aromatic Substitution in the Gas

Phase: The Importance of Fluoride Ion-Molecule Complexes Formed in Gas-Phase Reactions between

Nucleophiles and some Alkyl Pentafluorophenyl Ethers, J. Am. Chem. Soc. 104 (1982) 6520-6527. 
[8] W. Danikiewicz, T. Bienkowski, D. Kozlowska, M. Zimnicka, Aromatic Nucleophilic Substitution ( $\mathrm{S}_{\mathrm{N}} \mathrm{Ar}$ ) Reactions of 1,2-and 1,4-Halonitrobenzenes and 1,4-Dinitrobenzene with Carbanions in the Gas Phase, J. Am. Soc. Mass Spectrom. 18 (2007) 1351-1363.

[9] C. Hubert, H. Dossmann, X. Machuron-Mandard, J.-C. Tabet, ESI Formation of a Meisenheimer Complex from Tetryl and its Unusual Dissociation, J. Mass Spectrom. 48 (2013) 306-311.

[10] W. Danikiewicz, ,T. Bienkowski, D. Poddebniak, Generation and Reactions of Anionic $\sigma$-Adducts of 1,3Dinitrobenzene and 1,3,5-Trinitrobenzene with Carbanions in a Gas Phase, Using an Electrospray lon Source as the Chemical Reactor, J. Am. Soc. Mass Spectrom. 15 (2004) 927-933.

[11] S. Gronert, J.M. Garver, C.M. Nichols, B.B. Worker, V.M. Bierbaum, Dehalogenation of Arenes via $\mathrm{S}_{\mathrm{N}} 2$ Reactions at Bromine: Competition with Nucleophilic Aromatic Substitution, J. Org. Chem. 79 (2014) 11020-11028.

[12] G.J.C. Paul, P. Kebarle, Stabilities of Complexes of Br- with Substituted Benzenes (Sb) Based on Determinations of the Gas-Phase Equilibria $\mathrm{Br}^{-}+\mathrm{Sb}=\left(\mathrm{BrSb}^{-}\right.$, J. Am. Chem. Soc. 113 (1991) 1148-1154. [13] G.W. Dillow, P. Kebarle, Fluoride Affinities of Perfluorobenzenes $C_{6} F_{5} X$. Meisenheimer Complexes in the Gas-Phase and Solution, J. Am. Chem. Soc. 110 (1988) 4877-4882.

[14] J. Oomens, B.G. Sartakov, G. Meijer, G. Von Helden, Gas-Phase Infrared Multiple Photon Dissociation Spectroscopy of Mass-Selected Molecular lons, Int. J. Mass Spectrom. 254 (2006) 1-19.

[15] L. MacAleese, P. Maitre, Infrared Spectroscopy of Organometallic lons in the Gas Phase: from Model to Real World Complexes, Mass Spectrom. Rev. 26 (2007) 583-605.

[16] N.C. Polfer, Infrared multiple photon dissociation spectroscopy of trapped ions, Chem. Soc. Rev. 40 (2011) 2211-2221.

[17]B. Chiavarino, M.E. Crestoni, S. Fornarini, F. Lanucara, J. Lemaire, P. Maitre, Meisenheimer complexes positively characterized as stable intermediates in the gas phase, Angew. Chem. Int. Ed. 46 (2007) 19951998.

[18] B. Chiavarino, M.E. Crestoni, S. Fornarini, F. Lanucara, J. Lemaire, P. Maitre, D. Scuderi, Molecular complexes of simple anions with electron-deficient arenes: spectroscopic evidence for two types of structural motifs for anion-arene interactions, Chem. Eur. J. 15 (2009) 8185-8195.

[19] B. Chiavarino, M.E. Crestoni, P. Maitre, S. Fornarini, Halide adducts of 1,3,5-trinitrobenzene: vibrational signatures and role of anion- $\pi$ interactions Int. J. Mass Spectrom. 354-355 (2013) 62-69.

[20] B. Chiavarino, P. Maitre, S. Fornarini, M.E. Crestoni, Cyanide-Arene Meisenheimer Complex Generated in Electrospray Ionization Mass Spectrometry Using Acetonitrile as a Solvent J. Am. Soc. Mass Spectrom. 24 (2013) 1603-1607.

[21] J.M. Garver, Z. Yang, S. Kato, S.W. Wren, K.M. Vogelhuber, W.C. Lineberger, V.M. Bierbaum, Gas Phase Reactions of 1,3,5-Triazine: Proton Transfer, Hydride Transfer, and Anionic $\sigma$-adduct Formation, J. Am. Soc. Mass Spectrom. 22 (2011)1260-1272.

[22] B.P. Hay, V.S. Bryantsev, Anion-Arene Adducts: C-H Hydrogen Bonding, Anion-pi Interaction, and Carbon Bonding Motifs, Chem. Commun. (2008)2417-2428.

[23] Y. Song, R.G. Cooks, Atmospheric Pressure Ion/Molecule Reactions for the Selective Detection of Nitroaromatic Explosives Using Acetonitrile and Air as Reagents, Rapid Commun. Mass Spectrom. 20 (2006) 3130-3138.

[24] Y. Song, C.Y. Chen, R.G. Cooks, Reactivity of Acetonyl Anion with Nitroaromatics: an Atmospheric Pressure Chemical Ionization Study, Rapid Commun. Mass Spectrom. 19 (2005) 3493-3499.

[25] H. Chen, H. Chen, R.G. Cooks, Meisenheimer Complexes Bonded at Carbon and at Oxygen, J. Am. Soc. Mass Spectrom. 15 (2004) 998-1004.

[26] W. Danikiewicz, ,T. Bienkowski, K. Wojciechowski, Application of Electrospray lonization Mass Spectrometry for Studies of Anionic $\sigma$-Adducts of Aromatic Nitrocompounds, Tetrahedron Lett. 45 (2004) 931-934.

[27] J. Lemaire, P. Boissel, M. Heninger, G. Mauclaire, G. Bellec, H. Mestdagh, A. Simon, S. Le Caer, J.M. Ortega, F. Glotin, P. Maitre, Gas phase infrared spectroscopy of selectively prepared ions, Phys. Rev. Lett. 89 (2002) 273002-273004. 
[28] J.M. Bakker, T. Besson, J. Lemaire, D. Scuderi, P. Maitre, Gas-Phase Structure of a -Allyl-Palladium Complex: Efficient Infrared Spectroscopy in a 7 T Fourier Transform Mass Spectrometer, J. Phys. Chem. A 111 (2007) 13415-13424.

[29] M. A. Sainna, S. Kumar, D. Kumar, S. Fornarini, M.E. Crestoni, S. de Visser, A comprehensive test set of epoxidation rate constants by iron(IV)-oxo porphyrin cation radical complexes, Chem. Sci. 6 (2015) 15161529.

[30] C. F. Bernasconi, Kinetic Behavior of Short-Lived Anionic Sigma Complexes, Acc. Chem. Res. 11 (1978) 147-152.

[31] M.E. Crestoni, B. Chiavarino, V. Steinmetz, S. Fornarini, Communication: vibrational study of a benzyl carbanion: deprotonated 2,4-dinitrotoluene, J. Chem. Phys. 137 (2012) 181101.

[32] J.D. Steill, J. Oomens, Spectroscopically resolved competition between dissociation and detachment from nitrobenzene radical anion, Int. J. Mass Spectrom. 308 (2011) 239-252.

[33] I.L. Freriks, L.J. de Koning, N.M.M. Nibbering, Gas-Phase Ambident Reactivity of Acyclic Enolate Anions, J. Am. Chem. Soc. 113 (1999) 9119-9124.

[34] I. Fernandez, G. Frenking, E. Uggerud, Rate-Determining Factors in Nucleophilic Aromatic Substitution Reactions. J. Org. Chem. 75 (2010) 2971-2980.

[35] T. Giroldo, L.A. Xavier, J.M. Riveros, An Unusually Fast Nucleophilic Aromatic Displacement Reaction: The Gas-Phase Reaction of Fluoride lons with Nitrobenzene, Angew. Chem. Int. Ed. 43 (2004) 3588-3590. 